\title{
Mucosal Melanoma of the Head and Neck
}

National Cancer Institute

\section{Source}

National Cancer Institute. Mucosal Melanoma of the Head and Neck. NCI Thesaurus.

Code C133187.

A melanoma that arises in the mucosa of the nasal cavity, paranasal sinuses, oral cavity, oropharynx, nasopharynx, larynx, and hypopharynx. 